\title{
Analysis of variability in divergence and turn-over induced by three idealized convective systems with a 3D cloud resolving model
}

\author{
Edward Groot ${ }^{1}$ and Holger Tost ${ }^{1}$ \\ ${ }^{1}$ Institut für Physik der Atmosphäre, Johannes Gutenberg Universität, Johannes-Joachim-Becher-Weg 21, Mainz, Germany \\ Correspondence: Edward Groot (egroot@uni-mainz.de)
}

\begin{abstract}
The sensitivity of upper tropospheric and lower stratospheric convective outflows and related divergence fields is analysed using an ensemble of cloud resolving model (CM1) simulations in LES-mode including various physically manipulated simulations for three different convective systems initialized with an idealized trigger. The main goal of this study is to assess to what extend the divergence field depends on cloud microphysical processes, the mode of convection and on the processes of convective momentum transport and moist static energy redistribution. We find that latent heat release (representing the microphysical uncertainty) plays an essential role by explaining much of magnitude of the divergence field that will be formed. Convective organisation explains another important fraction of the variability in the divergence field that is formed by a convective system and behaves non-linearly, likely partly via condensation and subsequent (re-)evaporation/sublimation. The detrainment of stratospheric air also shows large sensitivity among the experiments.
\end{abstract}

\section{Introduction}

Error growth in numerical weather prediction models has widely been attributed to amongst others the simulation of moist convective processes (Tan et al., 2004), (Zhang et al., 2007), (Rodwell et al., 2013), (Selz and Craig, 2015b), (Selz and Craig, 2015a) and in the last years it has been considered as important model component to initially magnify error growth in NWP (Baumgart et al., 2019), (Bierdel et al., 2017), (Bierdel et al., 2018).

In Bierdel et al. (2017) a heat pulse produced in a hypothetical moist convective event is shown to excite geostrophic adjustment processes and a flow perturbation caused by convection is shown to propagate into balanced flow components with a theoretical model. The convective event is a source of heat (condensation) expanding the air in a convective cloud, which excites a density perturbation in the upper troposphere, from which a divergent flow is spun up. To better understand the role of individual convective events in this error growth processes, especially the divergence originating from convection, it is necessary to better constrain the causes of uncertainty in the representation of convection. For this purpose we conduct model simulations on the LES-scale, in which we vary individual processes in the convective development and analyse the impact on the divergence field, which as an ageostrophic wind component can substantially affect the downstream development of the wind field up to 
the synoptic scale. This uncertainty of the flow pattern represents an important source of uncertainty for weather prediction (Baumgart et al., 2019). An alternative approach is utilising a stochastic treatment of convection in ensemble weather forecasting (Selz and Craig, 2015b), which can similarly lead to an increased understanding of the variability of convectively imposed divergence.

In recent years many studies have been done to assess the representativeness and variability of convective circulations in cloud-resolving models, for example Petch (2006), Hanley et al. (2014), Stein et al. (2014) and Homeyer (2015). Petch (2006) formulated basic grid requirements for cloud resolving simulations with a case study, which are consistent with Bryan et al. (2003). Simulations have been statistically intercompared and also compared with radar observations in Hanley et al. (2014) and Stein et al. (2014). Results in Homeyer (2015) show that tropopause penetrating convective cloud tops and updraft intensity are very sensitive to grid length in WRF.

In this study the main purpose is to increase understanding of the sensitivity of idealized convective systems to various perturbations. Resulting divergent upper tropospheric (including lower stratospheric) outflow produced by a cloud resolving model (CM1) (Bryan, 2019) at resolutions down to 100-200 $\mathrm{m}$ is analysed. Three idealized cases of convection with two different initial buoyancy perturbations and exposed to two different wind profiles are simulated, with extra simulations to serve as an ensemble. With additional runs we explore sensitivity (i.e., modelled uncertainty) caused by the treatment of cloud microphysics (here highly idealized as latent heat release), vertical advection of horizontal momentum (convective momentum transport) and water vapor and how it affects the redistribution of moist static energy. The first factor (latent heat) is intrinsically unstable with convective instability and represents uncertainties in microphysical processes via condensation/evaporation in for instance droplet sizes and also in updraft buoyancy/acceleration. The latter is partly motivated with findings in numerical weather and climate models regarding the relevance of the parameterization of convective mixing of horizontal momentum, temperature and water vapor. Regarding advection of horizontal momentum it has been found that different convective equilibrium states could coexist, amongst others sensitively depending on the parameterized treatment of momentum exchange by convection (Nuijens and Emanuel, 2018), which may affect the flow and forecast propagation differently. The vertical advection of water vapor and temperature acts to reduce convective instability and hence determines how much the convection feedbacks on itself and from the downstream propagation point of view also affects the atmospheric state aloft notably. Hence we manipulate the vapor, temperature and momentum transports by convection to represent potential uncertainty in their strengths. However, the artificial disturbances of the convection are not supposed to be too strong to completely inhibit convection or lead to highly unrealistic updraft and downdraft velocities in an unrealistic physical state.

In the next section the methodology of this study is described, including the utilised model. Afterwards, the results from the individual simulations are presented and discussed, with a focus on the impact on the divergence field in the cloud vicinity. Finally, the results are interpreted with an outlook towards forecast uncertainty. 


\section{Methods}

\subsection{CM1 set-up}

Experiments have been conducted with cloud resolving model CM1 Bryan (2019). All simulations were done on a 120 by 120 $\mathrm{km}$ grid with a depth of $20 \mathrm{~km}$. The grid box size and shape have been varied. The reference simulation had grid cells of 200 $\mathrm{m}$ resolution in the horizontal and $100 \mathrm{~m}$ in the vertical. Additional simulations had $100 \mathrm{~m}$ and $200 \mathrm{~m}$ cubic grids and coarser grids of rectangles with aspect ratio 2.0. The model was run in large eddy simulation mode with time steps of $d t=0.75 \mathrm{~s}$ and the runs lasted two simulated hours each.

\subsection{Three cases}

Initially, a supercell simulation was implemented in accordance with Weisman and Klemp (1982), with the accompanied wind profile in CM1 ("high shear"), as displayed in Figure 1. Subsequently, a shallower sheared wind profile with less wind shear has been used (newly implemented), where the $u$ with $14 \mathrm{~m} / \mathrm{s}$ and $v$ with $4 \mathrm{~m} / \mathrm{s}$ in the $2500 \mathrm{~m}$ sheared layer ("moderate shear"; see also Figure 1). The vertical distribution of $u$ and $v$ have been set such that the model domain moves approximately storm relative, such that the residence time of moist convection (and its effects) within the model domain is nearly maximized.

The "supercell" experiment has been initiated with the default warm bubble setting (maximum strength $0.67 \mathrm{~K}$ at about $z=1.35$ $\mathrm{km}$ ) and "high shear". In addition, an ordinary multicell experiment has been done with the same warm bubble initiation and the "moderate shear" profile. As a final case, the second experiment ("moderate shear") has been re-initiated with a coldpool forcing (maximum strength $-6 \mathrm{~K}$ at surface level in the western half of the model domain), to trigger a squall line. All experiments have been simulated with each of these (see Figure 1) three case definitions as initial conditions.
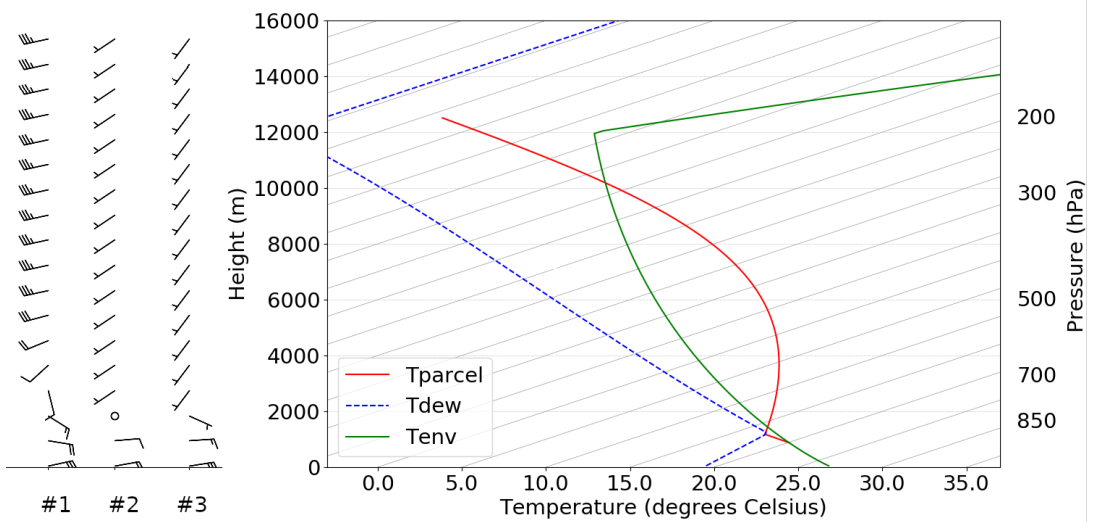

Figure 1. Skew-T diagram of the thermodynamic profile used, after Weisman and Klemp (1982). The red curve shows the evolution of a parcel with ambient conditions that is pushed upward from $z=875 \mathrm{~m}$ to its equilibrium level and beyond (without entrainment). On the left, the wind profiles used for the three cases (\#1: supercell, \#2: ordinary multicell, \#3: squall line, \#2 and \#3 with identical shear) are shown. 


\subsection{Overview of all experiments}

To map intrinsic sensitivity and uncertainty related to the reference experiment, an ensemble consisting of the reference run and 9 members has been used for comparison with subsequent experiments. Small perturbations (randomly generated, -5 to $+2 \%$, see Table $\mathrm{S} 1$ in supplement $\mathrm{S} 1$ ) have been applied to the altitude of the top of the shear layers $\left(z_{t o p}\right)$ in each wind profile to create initial conditions for each ensemble member.

Next, the model has been modified from a physical-dynamical point of view in the following ways for a set of experimental runs:

- Latent heat of vaporisation (lve) by $-40,-20,-10,+10$ and $+20 \%$, in order to alter latent heating, or analogously ambient stratification.

- Vertical advection of zonal and meridional momentum (VAUV) by $-100,-50,-20$ and $+50 \%$, altering mixing in the convective region.

- Vertical advection of water vapor (VAQ) (-20 and +20\%), altering energy (re-)distribution by the convective circulations.

All of the altered processes will initiate feedback loops and to some extent behave non-linearly due to an array of instability and restoration mechanisms that act in the atmosphere. (Therefore and) due to resulting chaotic behavior of the atmosphere we cannot fully disentangle the consequences (and also backward: root causes) of differences between experiments and cases.

No other settings than stated in 2.2 and this section have been altered with respect to the supercell simulation, which is included as a test case in CM1. The simulations have been run on Mogon2 in Mainz. For more details on the configuration and relevant files, see code availability. An overview of the experiments is also found in Table S1 of the supplementary material.

\subsection{Diagnostics}

(Horizontal) divergence has been calculated directly from the velocity fields in the output using finite differences on grid points. Moist static energy (MSE) has been calculated based on grid point values of potential temperature $\Theta$ and water vapor mixing ratio $r_{v}$ with a constant $C_{p}=1005.7 \mathrm{~J} \mathrm{~kg}^{-1} \mathrm{~K}^{-1}$ and (modified, but in principle constant) $L_{v}$ of $2.501 \times 10^{6} \mathrm{~J} \mathrm{~kg}^{-1}$.

$M S E \equiv C_{p} T+g z+L_{v} r_{v}=C_{p} \Theta+L_{v} r_{v}$

For the calculation of condensation rates, the CM1 output variable describing instantaneous condensation rate has been used. For the vertical advection tendencies we implemented a diagnostic in the modified CM1 code, without a divergence contribution (which would then be attributed to divergence rather than vertical advection, as discussed in Arakawa (2004)).

\subsection{Region selection for budget calculations}

A suitable area and time selection over which to integrate and subsequently average budgets of divergence, latent heat release, moist static energy, vertical advection of meridional and zonal momentum is done. To select a representative area affected by 
convective circulation and its (upscale) propagation, we include most of the region with gravity wave activity excited in the tropopause region (Figure 2). Triggered gravity wave activity reaches domain boundary within the two hour simulations, since convection initiates in the first hour and the waves propagate with up to about $30 \mathrm{~m} / \mathrm{s}$. The obvious effects from the model boundaries have been explicitly excluded as much as possible (Figure 3).

Since experimental aspects of the simulations make them not nearly identical at given time (except initially), there are some simulations in which the convection initiates earlier or later than in most other simulations, or the excitation and propagation of gravity waves differs from the ensemble. This leads to some inappropriate area selection for some individual runs: for underestimates in the area affected it removes part of the effects from the calculation. The actual effect in these runs will then be underestimated and typically lead to slightly less spread among the experiments, as the excluded effects make such experiments typically more dissimilar to other experiments than diagnosed by missing some effects.

\section{Results}

\subsection{Region of budget calculations}

As seen in Figure 2, the near-tropopause region at 11-12 km has the widest area of divergent flow induced by convection. Therefore Figure 3 displays the near-tropopause vertical velocity and the areas selected for the budget calculations for each of the three cases. The black box selections have been used to post-process all of the simulations. For cases \#1 and \#2 the areas are identical and both evaluated after 90 minutes of elapsed time. For case \#3 (squall line), the evaluation is done after only 75 minutes with a different area to limit effects from model boundaries (reflecting part of the waves). Both of the selected areas have nearly identical sizes (7080 vs. $7650 \mathrm{~km}^{2}$ ).

Other (both experimental and ensemble) simulations have been studied to check the applicability of the rectangular areas in Figure 3. In some experiments the area that is subject to gravity wave activity is slightly exceeding the rectangle for the supercell case. This is caused by ensemble sensitivity or by slightly different forcing (experimental runs), leading to slightly different initiation of convective activity and is intended to be taken into account while mapping the sensitivity. The magnitude of deviation from (similarity to) the ensemble hereby helps to interpret behavior of experimental simulations.

\subsection{Model resolution and case comparison}

In Figure 4 we see the vertical distribution of divergence, condensation rate, vertical advection of horizontal momentum (VAUV) and redistribution of moist static energy (MSE) averaged over the regions defined in Figure 3 and the previous section (3.1). In addition we see the ensemble with perturbed runs. As one would expect, a higher degree of organisation (supercell) and a larger area with convection (squall line) lead to larger amplitudes of all processes compared to the ordinary multicell case.

Obviously, the $1 \mathrm{~km}$ simulation, having fewer vertical levels, misses the finest details of the higher resolution runs, for example the peak magnitude of the near-tropopause divergence peak for the supercell and ordinary multicell cases. That means that the 


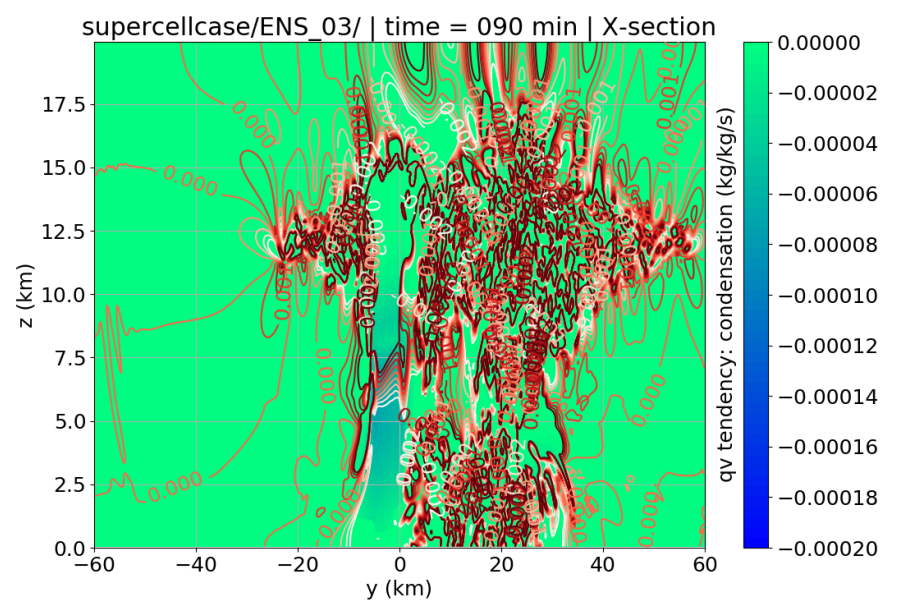

Figure 2. Condensation rate $\left(s^{-1}\right.$, colors) and convergence pattern in pink (divergence in brown) with isolines for each $5 e^{-4} s^{-1}$ after 90 minutes. This is ensemble member 3 of the supercell simulations, where the cross section (at selected $\mathrm{x}$ ) cuts through the cell core.
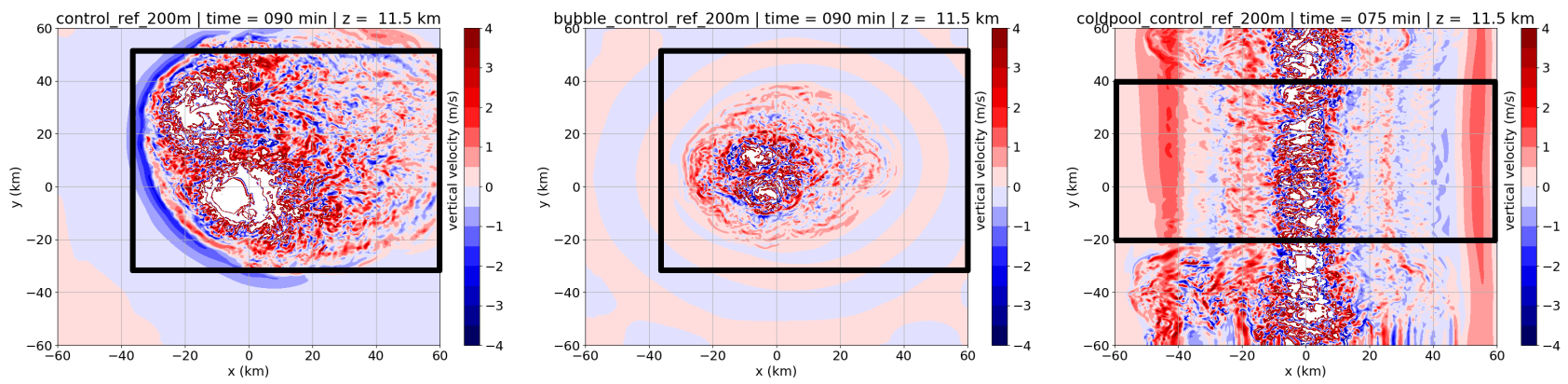

Figure 3. Vertical velocity near the tropopause in three runs (with absolute values $>4 \mathrm{~m} / \mathrm{s}$ left blank to emphasize the gravity waves) for three different cases in the reference run. Black rectangles show the areas selected for budget calculations of divergence and other quantities. Left: supercell, after $90 \mathrm{~min}$; center: ordinary multicell after $90 \mathrm{~min}$; right: squall line after 75 min.

$1 \mathrm{~km}$ simulation has not converged completely with the ensemble at higher resolution. The imperfect convergence of this $1 \mathrm{~km}$ simulation with the ensemble and missing of finest details in the vertical (reduced smoothness) is most obvious in the supercell case for the MSE and divergence profiles and the MSE profile in the squall line case. The condensation rates are lower in this case, which may have the same underlying reason. In the ordinary multicell simulations, vertical profiles seem to have shifted upward by one or a few hundred meters as well in the $1 \mathrm{~km}$ simulation.

Any case lacks substantial VUAV at $1 \mathrm{~km}$ and $500 \mathrm{~m}$ resolution, which is obviously because (explicit) momentum gradients are not fully resolved on these model resolutions. Additionally, we cannot determine from Figure 4 whether the cubic grid simulations and rectangular gridded reference simulation are sufficiently converged in terms of VAUV. 
Particularly the first two cases seem to have converged near the ensemble for the other quantities shown in Figure 4. With reference run and one other ensemble member slightly apart from the other eight the ensemble seems to contain a bimodal distribution in the supercell case, despite relatively strong convergence. However, the ensemble spread is much more pronounced and convergence is not reached for the squall line case.

The vertical distribution of divergence due to convection reveals patterns of convective outflow over 8-12 km/350 hPa to the tropopause, with a region of detrainment and mixing of lower stratospheric air (convergence) into the troposphere, where there is still a lot of (divergent) outflow above the tropopause at $12-14 \mathrm{~km}$ if convection is well organised (supercell and squall line). This is due to occasional tropopause overshooting cloud tops and it is also visible in the moist static energy redistribution curves. Accordingly lower and middle tropospheric convergence intensifies with a better degree of convective organisation. The MSE budget shows that both cases with warm bubble initiation (net) extract buoyant air from the boundary layer, as expected. Much of this air penetrates through the middle troposphere and ends up in the upper troposphere as a source of MSE. The MSE redistribution curve displays the detrainment of stratospheric air mixing slightly warmer air into the troposphere well. For the supercell case the profile has again slightly stronger amplitude than for the ordinary multicell case, as expected. In the squall line simulations MSE is net hardly extracted from the near surface layer, but rather from just above the boundary layer. Upon inspection of the vertical MSE cross sections perpendicular to the orientation of the squall line (not shown), it is found that even though the near surface layers are a source of buoyant air and thus locally of MSE in this case, the downdrafts initially cause a small MSE increase in the forward flank of the coldpool locally and gravity waves also cause some downward mixing of higher MSE that compensates the small area from which moist static energy is depleted. In addition the simulation domain moves storm relative, which with easterly low level winds means that potential source air (high MSE) is ingested at model boundaries. Nonetheless the MSE gain in the middle and upper troposphere is deeper and has more amplitude as in the other two idealized cases, while the stratospheric extraction of MSE seems comparable to that in the supercell simulations.

\subsection{Latent heat release experiments}

In Figure 5 the result of simulations with modified constants of latent heat of vaporization (lve) are shown. Roughly speaking, an intensification of convection is seen with increasing (lve), which is obviously related to higher values of buoyancy and CAPE with more latent heating. The vertical outflow levels are obviously increasing with latent heating and colliding increasingly strongly with tropopause in the supercell case for simulations with lve $>100 \%$ and consequently the divergence is affected accordingly. In accordance with vertical outflow level and magnitude, size of convective cells also clearly correlates positively with increasing lve and more buoyancy and condensation (not shown).

In $-40 \%$ latent heat constant runs, CAPE is strongly reduced for each case to slightly above $200 \mathrm{j} / \mathrm{kg}$ and all of them have much smaller and shallower convective clouds, hardly affecting the near tropopause region. Minor effects on the quantities of interest near the tropopause are visible just for the squall line. In this simulation, moist static energy is net not extracted from the boundary layer, but ingestion of air at the eastern boundary is strong and along with downward mixing of MSE by gravity waves in the wake of the squall line, the net gain in MSE in the lower layers $(z<2.5 \mathrm{~km})$ can be explained. 

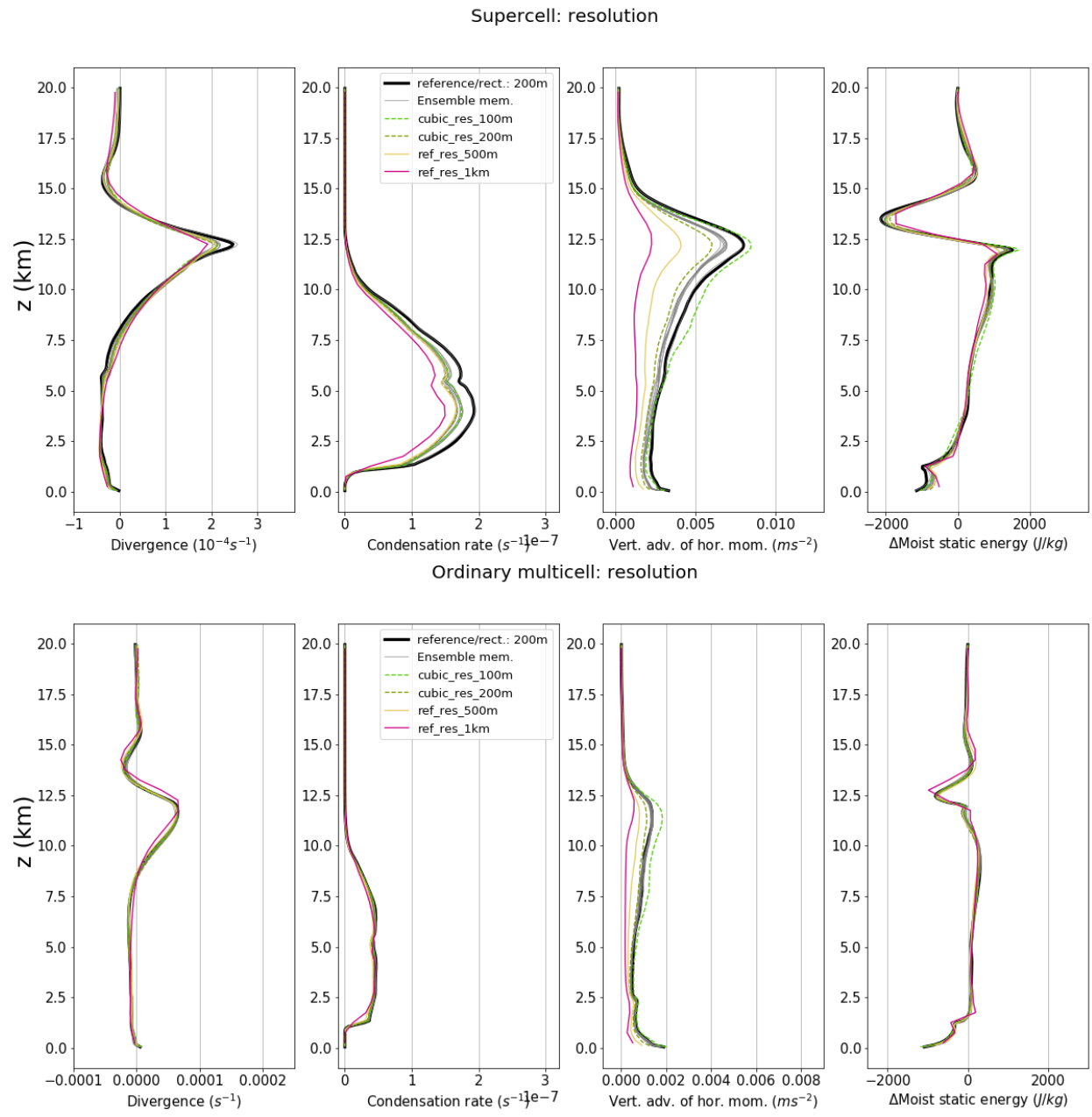

Squall line: resolution
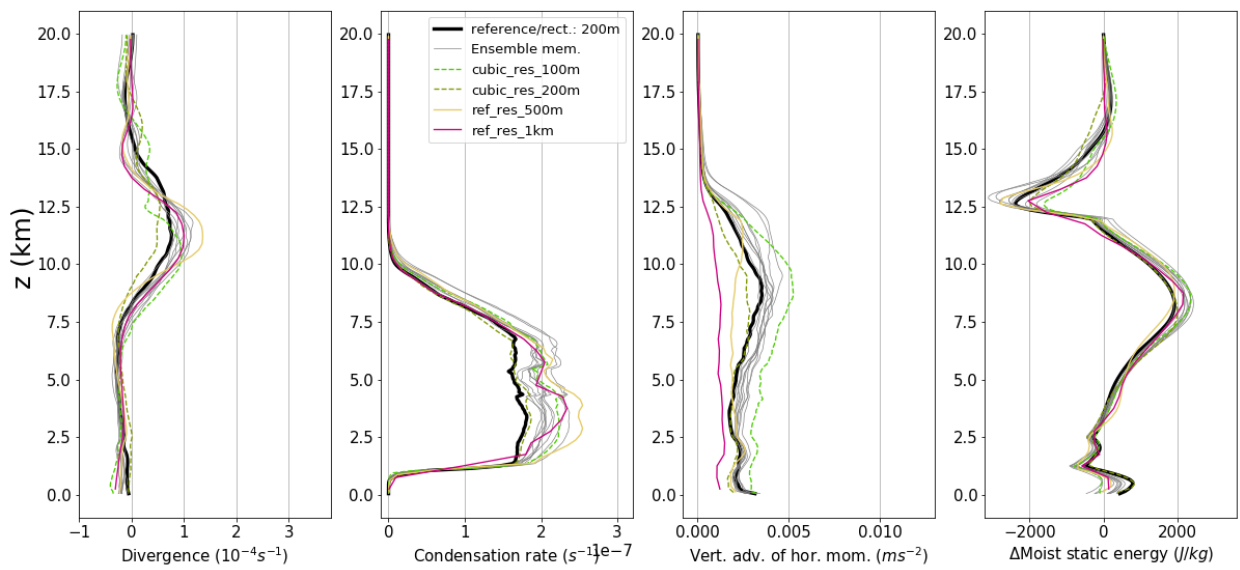

Figure 4. Vertical display of horizontally integrated budget profiles of divergence at time $t$, mean condensation rate up to $t$, mean VAUV up to $t$ and $\triangle \mathrm{MSE}$ up to $t$. Here the resolution experiments at five different resolutions and the ensemble are shown (reference run in black).

Top: supercell simulations; center: ordinary multicell simulations; bo 
of the $-40 \%,-20 \%$ and $+20 \%$. In case of ordinary multicell convection, all runs are significantly different from the ensemble, while this is less pronounced for the squall line case due to larger ensemble spread.

Particularly strong non-linearity is seen in stratospheric detrainment of high MSE values for the ordinary multicell experiment. This mixing may potentially significantly affect (prognostic) thermodynamic profiles at near-tropopause level. the latent heat constant with a given amount than the squall line. This is probably due to the stronger forcing of convection when it is initiated with a coldpool in the western half of the domain. It is suggested that forcing and subsequent mode of convection/convective organisation affects the way and magnitude at which convection modifies the atmosphere and subsequently the flow field in different non-linear fashions, given an ambient stratification profile. In other words: how feedbacks between dynamics and the intensity of convective circulations are affecting larger scale flow depends on mode of convection at a given convective instability. We will further explore such non-linearity in Section 3.6.

\subsection{Momentum advection experiments}

It is generally accepted that modification of VAUV affects convective organisation. First of all the genesis of a supercell requires this term in the momentum equations: it is necessary to tilt horizontal vorticity. The interaction of this tilted vorticity with updraft dynamics is necessary for supercell formation Markowski and Richardson (2010). Furthermore, absence of the term in the ordinary multicell and squall line cases limits the role of coldpools in the propagation of the convective system and, in relation to this, limits the tilting of warm air bubbles over the coldpools that feed convective cells. In other words: the easterly inflow east of the squall line cannot be brought upward into the squall line for instance and lead to the typical flow pattern in a squall line Markowski and Richardson (2010). Therefore all VAUV experiments have (strongly) modified convective structures and areal sizes compared to the reference runs.

Some interesting consequences of amplified and suppressed VAUV can be identified in Figure 6. Non-linearities affect the diagnosed VAUV (see also Section 3.6). VAUV itself adjusts strongly at near tropopause level if non-zero and near the surface (+50\% VAUV run). This can partly be explained by VAUV peaking around these two levels.

Condensation rates (Figure 6) are either reduced notably (0.0, 0.5 and 1.5 simulations for supercell case, 0.0 and 0.5 simulations for the squall line case and 0.0 for the ordinary multicell case) or weakly affected (other simulations). Only the squall line case leads to extra condensate at $+50 \%$ VAUV, but this falls within the ensemble range. In other words, the reliable momentum advection scheme seems to nearly maximize condensate and supports efficient convective turn-over. Part of the adjustments in condensation profiles are explained by changes in organisation of convection and the area with moist convection, described earlier in this section.

The supercell case shows that the ensemble members are accordingly most efficient regarding MSE turn-over, with particularly pronounced weakening for no and -50\% VAUV. Among the ordinary multicell simulations spread is small for varying VAUV. For the squall line case, MSE exchange grows monotonically with increasing VAUV, consistently with what is seen in the condensation rates.

Divergence effects resemble those of area mean condensation rates. Generally, adjusted VAUV experiments also tend to dis- 

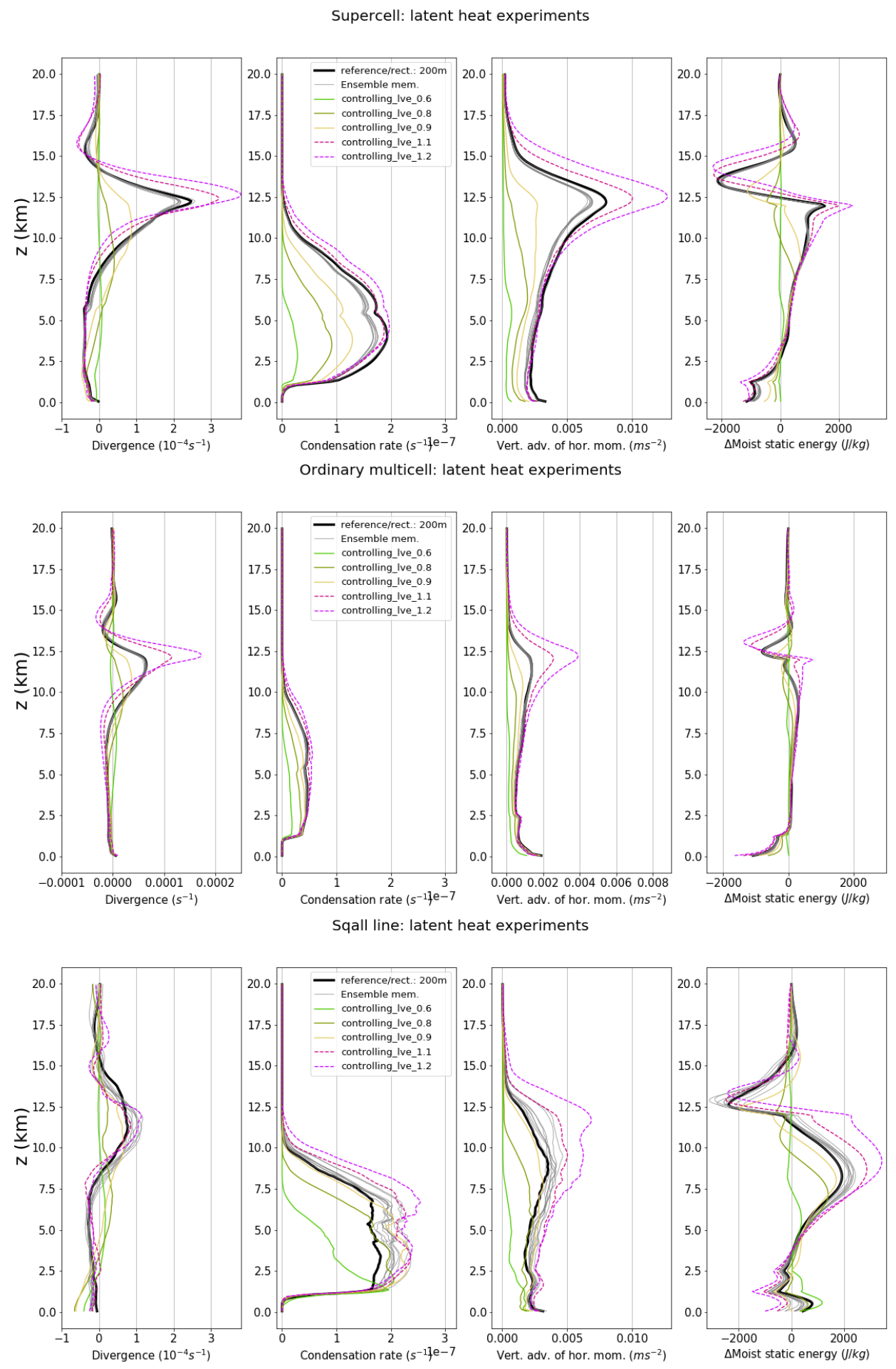

Figure 5. See Figure 4. Here the lve experiments and the ensemble are shown, with suppressed latent heat constants indicated with a solid line and enhanced latent heat constants with a dashed line. Top: supercell simulations; center: ordinary multicell simulations; bottom: squall line simulations. 
tribute the upper tropospheric and lower stratospheric divergence $(>0)$ over a layer that starts slightly lower, with a smaller peak amplitude. Most modified VAUV runs (except squall line with $+50 \%$ VAUV) fall with their divergence outside the ensemble range in the upper troposphere and lower stratosphere, which means that momentum mixing by convection has a significant effect on the divergence field locally and particularly on the peak value.

\subsection{Water vapor advection experiments}

215 In Figure 7 the budgets of simulations with modified vertical water vapor advection are depicted. An important characteristic of the 0.8-run that can be found in all three cases is the occurrence of condensation at near-surface levels, as opposed to all other runs so far. This is because horizontal moisture convergence cannot be completely compensated by upward transport of water vapor before condensing, leading to near-surface hydrometeor accumulation by the convergent flow. This means that the run has physical behavior that is not realistic. The condensation profiles for this run are shifted downward, probably due to the aforementioned effect. The magnitude of total condensation is affected both negatively and positively in the modified water vapor advection runs, depending on the case. This is explained by total area of convective clouds in the three different cases: the supercell becomes smaller with less condensation, while the ordinary multicell shows more condensation and a larger area of convective cells.

With amplified vertical advection of water vapor, we can see strong non-linear increases in condensation, which occur because once a convective updraft establishes it is vertically more permeable to moisture than in the reference run. This also manifests in the simulations by increasing the size of convective clouds and coldpool strengths. Basically, both amplified and reduced vapor advection can have a qualitatively similar effect on amount of vapor condensation. The water vapor advection example thus clearly illustrates some of the non-linearity of the experiments.

The effects of the 0.8-runs compared to the reference runs regarding the VAUV in cloud regions are seemingly non-linear and correlated to the condensation effects slightly below, with enhancements in condensation leading to higher VAUV and decreased condensation leading to lower values of VAUV. Likewise, the reversed happens when vertical vapor advection is amplified, with momentum mixing increasing throughout the depth of the convective circulation. Many of the divergence effects resemble those seen in VAUV and are therefore similarly related to the condensation rates slightly below.

In principle, there is a direct effect of amplified (reduced) vertical vapor advection on MSE change, i.e. more water vapor advection increases MSE transport and change. Feedbacks such as consecutive phase changes can overcome the intuitive effects and reverse or enhance the net modification in MSE change and subsequently also for divergence. There are clearly non-linear amplification effects in the 1.2-run that impact MSE redistribution and divergence (also evident effects in the condensation profile). On the other hand, the 0.8-run has counter-intuitive/reversing effects in the ordinary multicell case: net divergence is increasing rather than decreasing compared to the reference run, which is related to increased condensation. MSE redistribution is also not clearly weakened. Strong sensitivity to vertical water vapor advection is found in the detrainment of stratospheric air near the tropopause, like in other experiments - most clearly in the ordinary multicell and squall line cases. Additionally, the divergence profile of the 0.8-run with squall line clearly deviates from all other runs. The divergent convective outflow peak falls from 10-13 to 6-10 km, which is partly because cell penetration of the tropopause is delayed (not shown) and the squall 
Supercell: momentum advection experiments
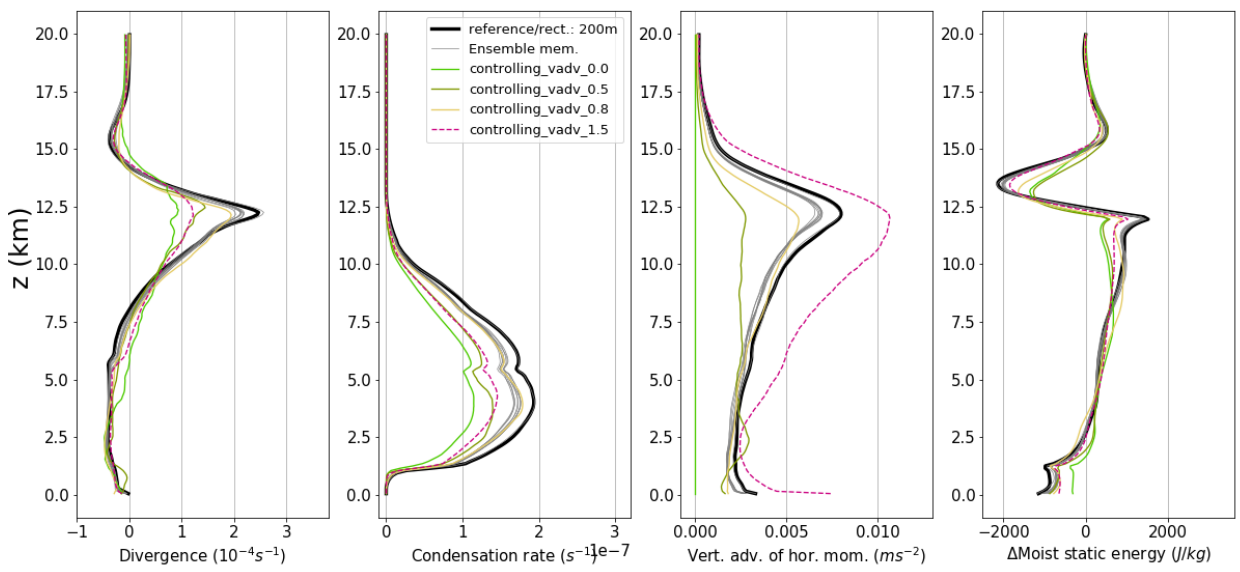

Ordinary multicell: momentum advection experiments
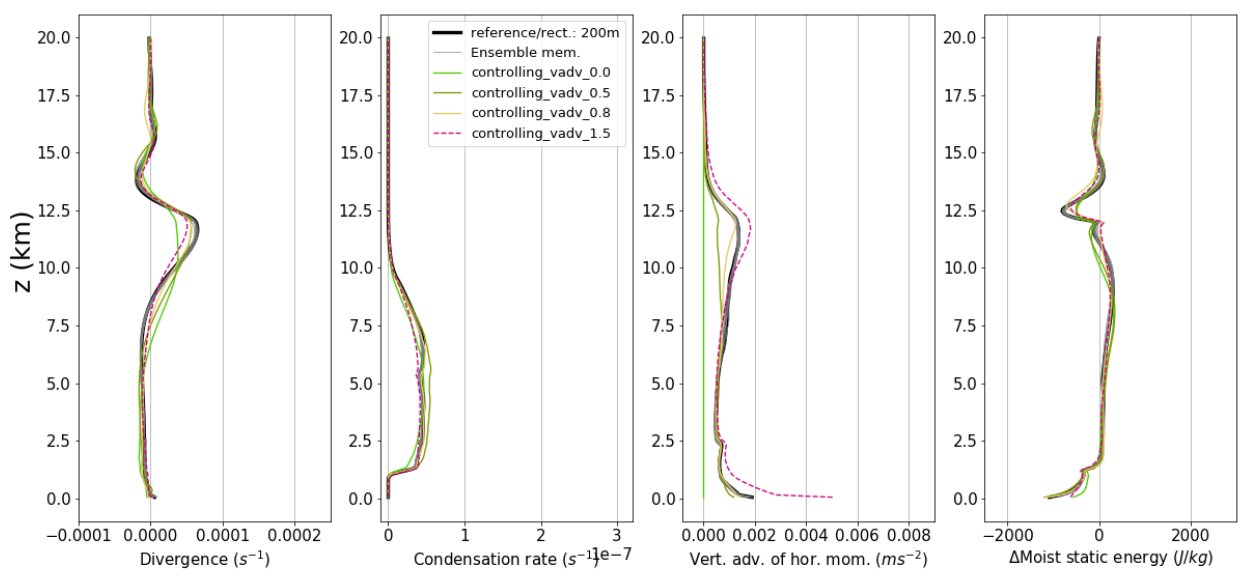

Sqall line: momentum advection experiments
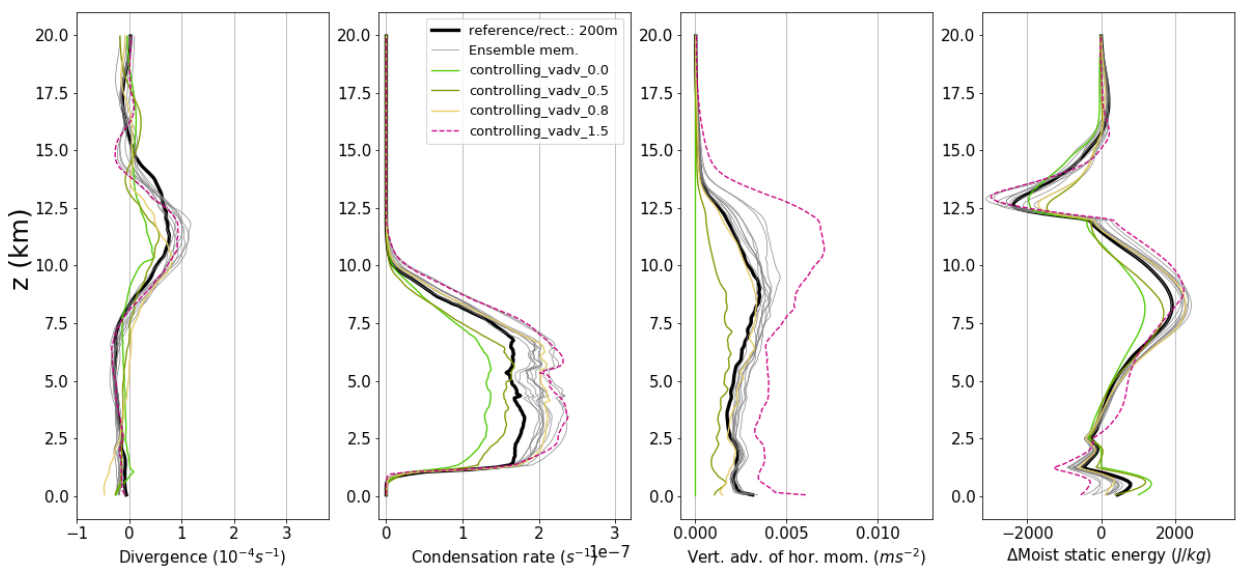

Figure 6. See Figure 4. Here the VAUV-experiments and the ensemble are shown, with supressed advection indicated with a solid line and enhanced advection with a dashed line. Top: supercell simulations; center: ordinary multicell simulations; bottom: squall line simulations. 
line budgets are analysed after 75 minutes, earlier than for the other two cases. While in other simulations net convergence generally still occurs up to around 7-8 km elevation (see Figures 4-7), this specific simulation has its divergence peak around that level. Therefore, the net elevation where the convergent inflow occurs, sinks substantially to below $3 \mathrm{~km}$ and convergence below that level intensifies substantially to conserve mass.

\subsection{Feedback strengths}

In Figure 8 some feedback strengths are visualised. The quantities on the y-axis are averaged over the 100-300 hPa layer in this plot and weighted by pressure, i.e. a model layer with more mass is weighted accordingly. In the plot, the $(1,1)$-points are reference simulations and the (0,0)-point would mean no latent heat release and no divergence in Figure $8 \mathrm{a}$. It can be seen in Figure 8a that the outflow of convection and its divergence in the 100-300 hPa layer is very sensitive to changes in the latent heat constant or equivalently to an altered ambient stratification (see also Figure 5). Furthermore, the squall line simulation behaves differently in terms of feedback strength than the other two cases of isolated convection, with no mass weighted divergence increase beyond the reference simulation in the selected layer for $+10 \%$ and $+20 \%$ lve. It should be mentioned that the upper tropospheric and lower stratospheric divergence in convective outflows is sensitive to the layers included in the analysis. When the same computations are plotted over the 400-100 hPa layer for example, the curve would flatten off between 1.1 and 1.2 instead of rising to nearly 1.6 for the isolated convection cases (not shown).

It is also interesting to notice that the ordinary multicell has a negative divergence contribution for a $-40 \%$ latent heat constant, which is because the divergent outflow is located below $300 \mathrm{hPa}(z=9.3 \mathrm{~km}$, see Figure 5). Therefore, detrainment from around 200-300 hPa causes net downward mixing and convergence in the layers used for Figure 8a. For the other two cases, little happens in the considered layer at a $-40 \%$ latent heat constant.

In Figure $8 \mathrm{~b}$ it can be seen clearly that actual amplification of VAUV is non-linearly reacting to amplification factors imposed on it, as described in Section 3.4. The actual amplification factors are clearly dependent on mode of convection and near-linear for the supercell and ordinary multicell simulations, but not for the squall line simulations, where we can see a lot of variability among the ensemble members.

Figure 8c shows that eliminating VAUV generally reduces divergence in the outflow layer. While increasing the VAUV, the divergence at first increases, but trajectories via which it increases are variable. The sensitivity of divergence to VAUV is clearly significant (Figure 8c) and suggested to be substantial in well organised convective systems. Since the feedback strengths presented in Figure 8 are depending on many factors, it is not meaningful to generalize much more.

\subsection{Divergence imposed by convective systems}

In Figure 9 the relation between density weighted mean of the divergence over the 6-14 km layer and total condensational heat is shown. The supercell and ordinary multicell convection seem to behave differently than the squall line case. Among the idealized supercell and ordinary multicell convection there is a good coherence between this upper tropospheric (+ lower stratospheric) divergence and condensational heat, whereas the idealized squall line case has an array of ratios between the two (scattering the plot) with less divergence per unit latent heat release than the other two cases. This is likely partly explained by 
Supercell: water vapor advection experiments
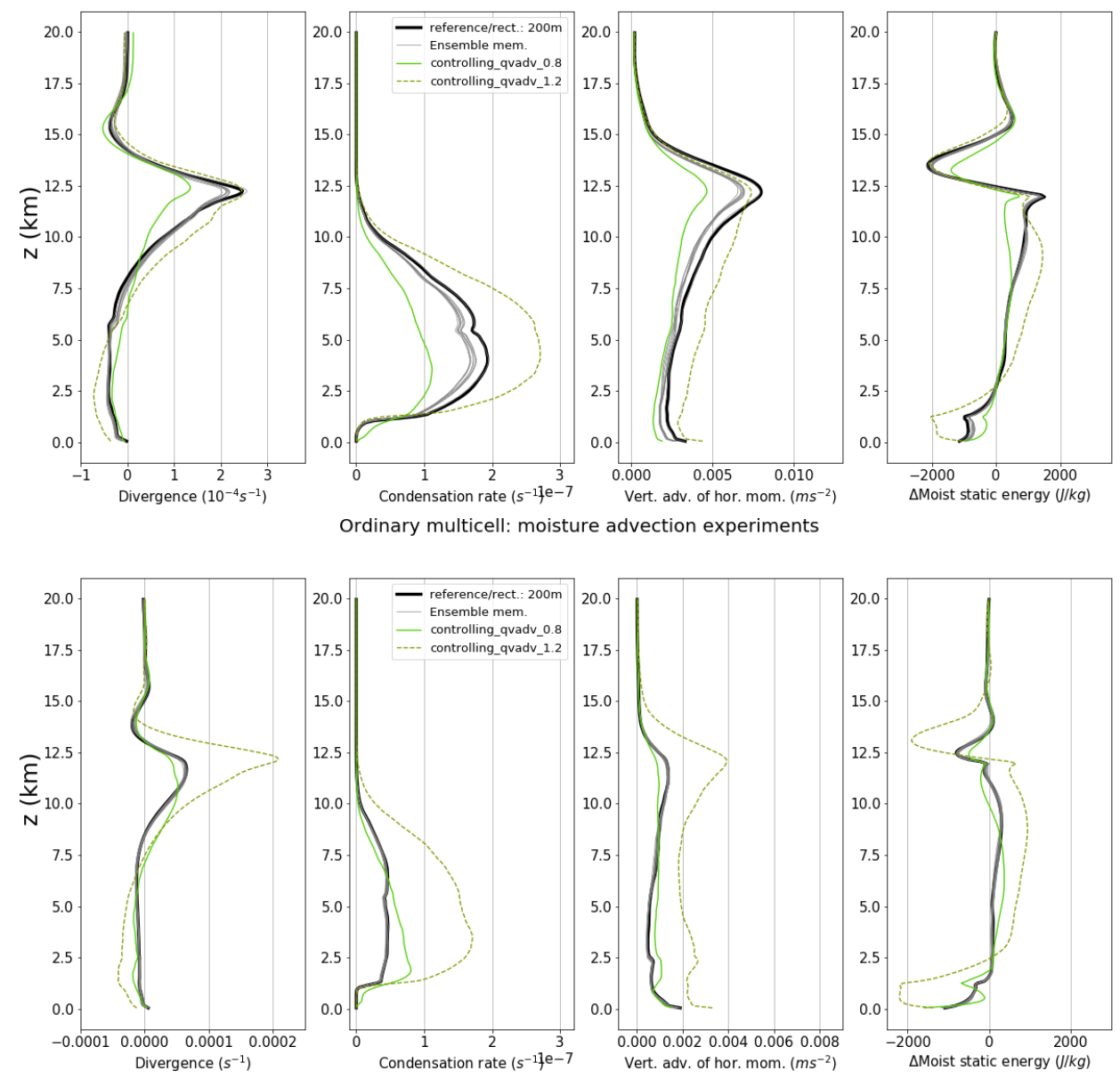

Squall line: water vapor advection experiments
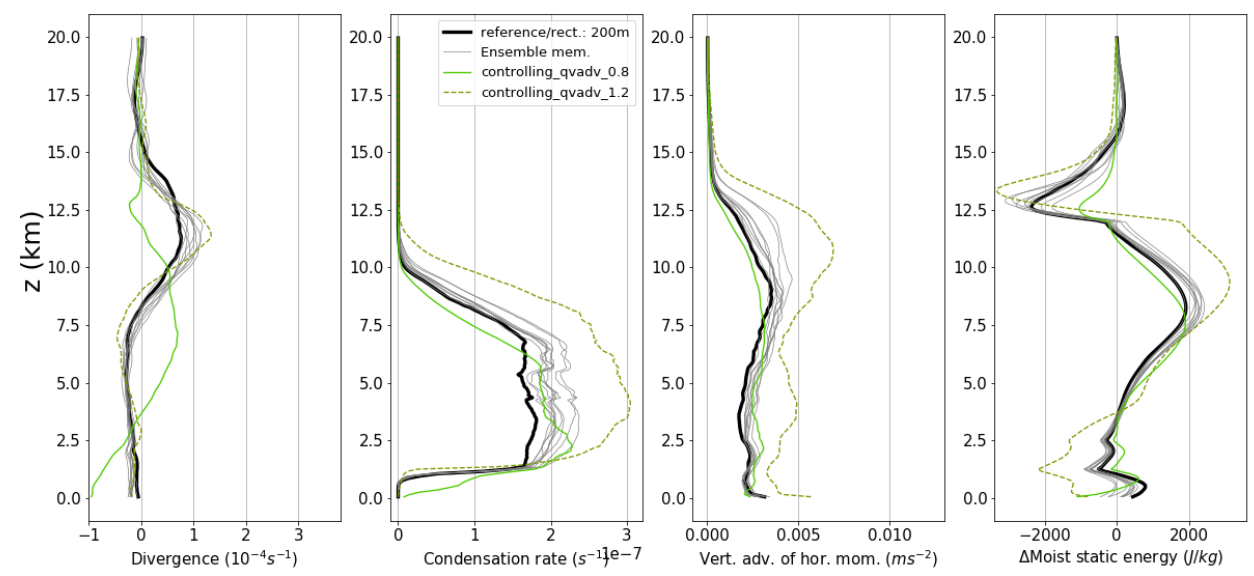

Figure 7. See Figure 4. Here the vertical moisture advection experiments and the ensemble are shown, with supressed vertical moisture advection indicated with a solid line and enhanced vertical moisture advection with a dashed line. Top: supercell simulations; center: ordinary multicell simulations; bottom: squall line simulations. 

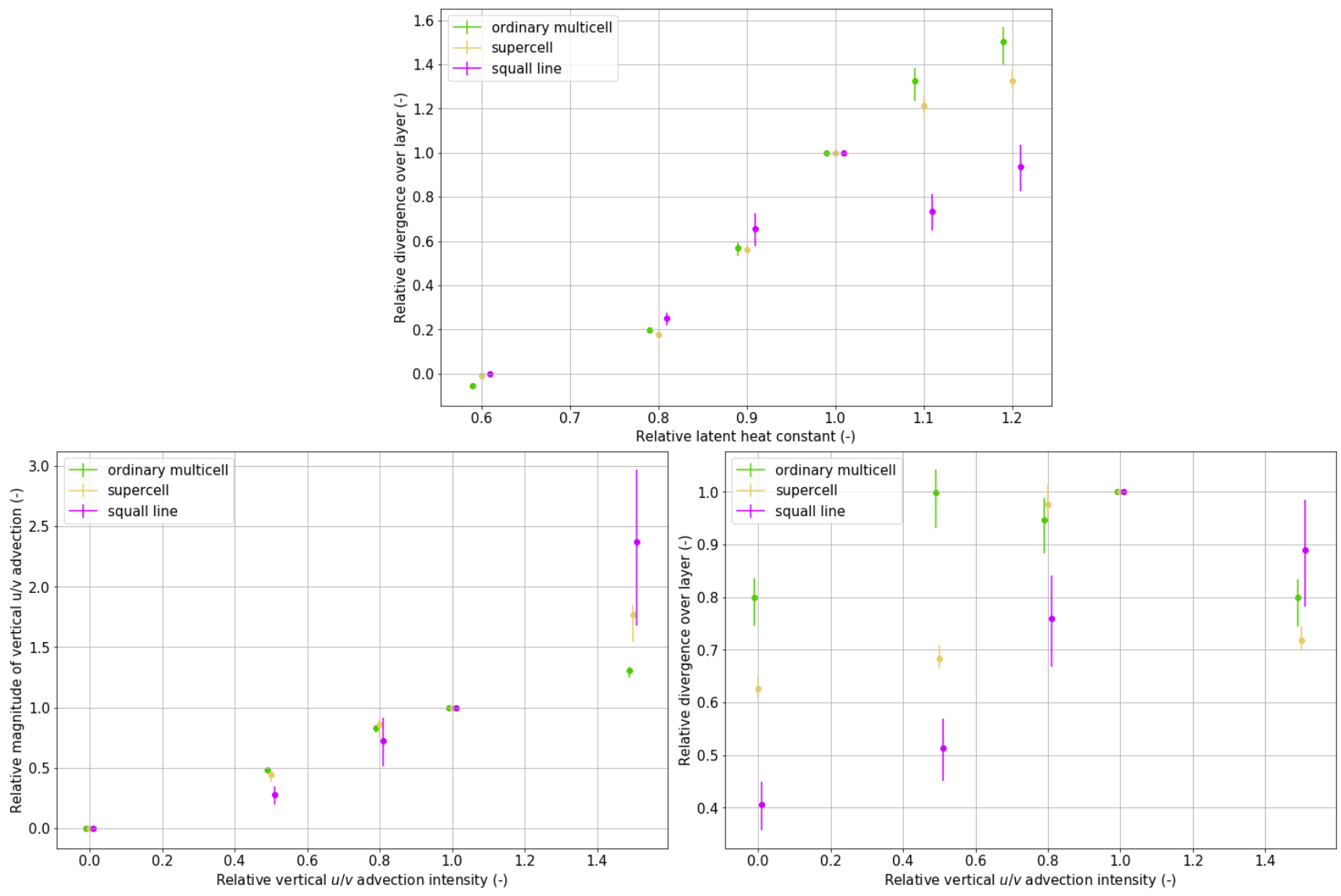

Figure 8. Feedback strengths found in the experiments over 100-300 hPa layers (pressure weighted), normalized by reference runs (1,1). Length of the error bars in experimental runs indicate the maximum and minimum values for the individual ensemble members and the point marker indicates the ensemble mean. Top: Latent heat constant on divergence; left bottom: imposed VAUV amplification on the diagnosed VAUV; right bottom: imposed VAUV amplification on divergence.

the recycling of condensate: out of all condensed mass in the squall line runs subsequent evaporation or sublimation happens for 0.32-0.40, whereas in the supercell and ordinary multicell simulation only for a smaller fraction, typically 0.19-0.23 and 0.25-0.31. The fractions are clearly higher than those mentioned above exclusively for the experiments with the lowest value of latent heat constant (-40\%). Nonetheless, the full variability in Figure 9 cannot be governed linearly by just aforementioned fractional differences in recycling of water vapor, as Figure 9 shows very roughly twice as much divergence per unit condensational heat in squall line simulations than in the other two cases, while the recycling of condensate deviates only slightly between the two.. Therefore other quantities must feedback on the relationship shown in Figure 9.

The inflow and outflow height of convective cells would also be expected to affect the relationship shown in Figure 9. However, apart from the control_lve_0.6 and to a lesser extent control_lve_0.8 (see Figure 5) these heights are relatively similarly distributed in the vertical for all cases. Since the control_lve_0.6 and control_lve_0.8 runs for the supercell and ordinary multicell 
case are not clearly far off the general relation in Figure 9, inflow and outflow height can also only explain a (very) limited fraction of the variability in the relationship.

Another reason for the deviating relationship of the squall line case from the other two could be the (efficiency of) bringing the middle and upper troposphere closer to equilibrium by the convection. Figures 4 to 7 signify that simulations of the squall line case generally bring much more MSE to the 6-10 km layer than simulations of the other two cases. This leads to buoyancy perturbations that should decrease more strongly in time and herewith the expansion of air in the convective updraft would be limited. It should be noted however, that the potential temperature perturbations in the convective cells with respect to the bulk at about $7.5 \mathrm{~km}$ height after 75 minutes are found to be similar to that for the ordinary multicell case if corrected for total area of convective cells, but the perturbations deviate for the supercell case. This would suggest that relaxation of the instability is probably not important for the deviating squall line simulations.

Another way to explain the deviation between squall line and other simulations in Figure 9 would be starting from the differing forcing to initiate convection, as this was a manually imposed difference between the squall line and other cases. From that point of view it is unlikely that the full chain of causes is tracked so far in this section, so it remains an open question what additional behavior is responsible for the relation in Figure 9.

If the depth over which Figure 9 budgets are integrated is somewhat modified (e.g. $9-15 \mathrm{~km}$ or $5-14 \mathrm{~km}$ ), the pattern of scattering is largely conserved and the individual experiments move slightly. Relations between one another are thence largely conserved. Note that the vertical layer of interest for our research question about deep convection stretches definitely from about 5-7 km to the lower stratospheric detrainment layer where convective clouds may end up after overshooting the tropopause and therefore the signal in Figure 9 is robust.

In Figure 9 we can discriminate between more realistic and less realistic behavior of convection in a simulation. Despite the strong and very idealized forcing of convection in our three cases which is not very realistic, the deep convection can be assumed to be simulated most realistically with grid cells of size 100-200 m. The coarser grid simulations will behave somewhat less realistically, as much of the turbulence is for example still parameterized. This can be seen in the ensemble and resolution experiment of Figure 4 (VAUV). Additionally, the ensemble and latent heat experiments will behave most realistic, as opposed to the experiments with modified momentum and moisture advection, which violate laws of conservation. Explicitly violating laws of conservation leads to impossibly strong build up of buoyancy and CAPE in some simulations for example, with very localized high CAPE patches far beyond $4000 \mathrm{j} / \mathrm{kg}$.

The most reliable blue and yellow markers in Figure 9 demonstrate that there is likely substantial variability of upper tropospheric and lower stratospheric divergence in response to condensation occurring in convective clouds in the real world. Substantial variability is also found in the vertical distribution of the divergence (Figures 4 to 7 ). Some of the variation can be attributed to the amount of condensate and some of it probably to the mode of convection, with linear systems likely being less efficient and more variable in their efficiency to convert condensate into upper tropospheric and lower stratospheric divergence. 


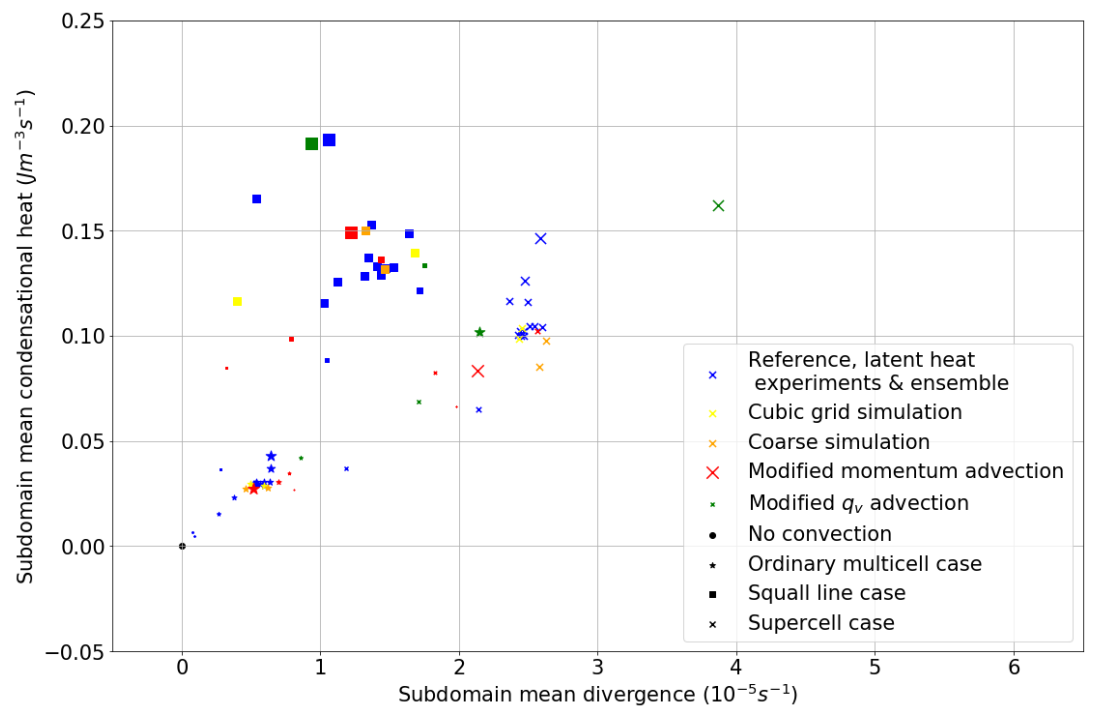

Figure 9. Relation between divergence over the 6 - $14 \mathrm{~km}$ layer (density weighted) in the runs and total latent heat release over the model depth until the budget evaluation and horizontally integrated over the budget evaluation area. The symbol size indicates multiplication factors used for each run, e.g. the smallest blue symbol has a latent heat constant of $-40 \%$ compared to the reference run.

\section{Discussion}

\subsection{Context}

\subsubsection{Accuracy of simulation of convection and impact on large scale processes}

Our results suggest that the simulation of organised convection and its divergence field affecting ambient flow depends model resolution, with higher resolution simulations probably accounting better for the processes involved in convection, arguing that turbulence is more explicitly resolved with higher resolution simulations. This is also argued by Bryan et al. (2003). Our study is nonetheless fully a modelling study and additional support (involving observations) for this line of argumentation can be found in Stein et al. (2014) and Hanley et al. (2014). In Stein et al. (2014) it is argued that convective storm structures in a case study over Southern-England converge for simulations at resolutions of 200-100 m with the UK MetOffice model, which is indeed consistent with Bryan et al. (2003), and these converged high resolution simulations represent statistics of three dimensional convective cloud structures in radar detections better than a $1500 \mathrm{~m}$ resolution simulation. Despite the former, high resolution simulations tend to have small convective cores with an overestimate of precipitation intensity. In Hanley et al. (2014) this is further elaborated: higher resolution models perform better in simulating convection down to scales below a radius of about $3 \mathrm{~km}$, but while also varying the subgrid mixing length the correct statistical representation of cell size and precipitation intensity is never achieved simultaneously for the two considered cases. Likewise, it is possible that the representation of the full variability of mean upper air divergence and mean condensational heating rate (Figure 9) is not 
achieved in our experiments, despite the wide range of experiments we did.

In Peters et al. (2019) it is argued that the effect of mesoscale convective systems on the ambient large scale flow is represented much better in models with (partially) resolved convection than in models with parameterized convection over Western Africa. Consistently with our results, higher resolution simulations clearly induce a stronger upper tropospheric divergence above mesoscale convective systems. However, they compared a month of one day convection resolving simulations (resolution 2.5 $\mathrm{km}$ ) with hydrostatic simulations in which convection is parameterized (resolution $30 \mathrm{~km}$ ) over a much larger domain. In addition to the identification of regime-like behavior by Nuijens and Emanuel (2018), such behavior of convective systems has recently also been explored with a many simulations approach (like ours) by Lawson (2019). Very strong indications of regime and tipping point behavior other than the actual formation of convection itself were found, identifying reflectivity objects as convective elements. Our results can complement this study, in which intervariability among convection in nearly similar environments has also been tested, but none of the experiments had a (very) strong forcing. Our squall line experiments suggest that regime behavior may exist in a forcing continuum as well, among the many degrees of freedom that atmospheric convection has.

\subsubsection{Ensemble perturbation saturation}

One might wonder whether the initially tiny perturbations actually saturate to the intrinsic variability and the envelop of samples that is statistically of interest with our initial conditions: in other words whether the predictability at the end of the run vanishes and a full (non-linear) regime is appropriately sampled. The applicability of our ensemble can be checked regarding that question. Quantitatively a statistical estimate can be made to address this applicability problem.

To illustrate non-linearity and saturation of twin (perturbation) experiments, Hohenegger and Schär (2007) use a correlation measure: from two initially opposing temperature perturbations (equal, but one of negative and one of positive sign) in their ensemble, Hohenegger and Schär (2007) subtract a reference run temperature to obtain two difference fields (as a function of time). Then, they compute the evolution of the correlation of these two difference fields in time. They also derive that starting from - 1 , the correlation coefficient between the two difference fields should approach 0.5 after the errors become random fields that saturate as a consequence of non-linear behavior (Hohenegger and Schär, 2007) (derived in their appendix). We have followed a similar procedure for $u$ (perturbed in our ensemble): we made pairs and derived difference fields with a common reference. Since our ensemble is randomly and non-monotonically (monotonically, except variations in $z_{\text {top }}$ ) perturbed (see 2.3 and Table S1 (supplement) includes the exact numerical values of perturbations), the correlation coefficient can start both near -1 and +1 , but not exactly at these values. All of the 12 evaluated pairs except one show similar behavior: difference fields start near \pm 1 , are nearly constant during the first 10-25 minutes and then quickly move to a value near +0.5 , reach this value after about 40 minutes and stay particularly close to +0.5 from 70 to 115 minutes ( $t=120$ minutes was not evaluated). The sole exception is the squall line case ensemble 1 and 9 pair due to a near-identical random perturbation (see Table S1: ENS_01 \& ENS_09), such that they have initially a nearly perfect correlation and diverge to correlations of 0.66-0.71 during the 70-115 minutes interval. The correlation plot for all pairs is shown in (Figure) S2 of the Supplementary material. The behavior suggests that the ensemble is not underdispersed and suitably examines the uncertainty margins we are interested in. 


\subsection{Summary \& outlook}

We have tested the sensitivity of upper tropospheric and lower stratospheric convective outflows and corresponding divergence fields for three different convective systems initialized with an idealized trigger. This was repeated with as an ensemble experiment and with various manipulated simulation settings to improve understanding of the generated divergence and its sensitivity and to assess the relationship with the amount of condensate and some feedbacks. The overall goal was to explore to what extend the divergence field depends on latent heating, convective organisation and on the processes of convective momentum mixing and water vapor/moist static energy redistribution.

Our experiments with cloud resolving model CM1 demonstrate that there is substantial uncertainty in upper tropospheric and lower stratospheric divergence fields arising from idealized cases of deep convection, particularly for the idealized squall line simulations (larger ensemble spread). The three cases demonstrate that uncertainty in latent heat release (which can be understood as level of detail in the convective cloud microphysics (especially in parameterised convection) plays an essential role by inducing the shape and explaining well the magnitude of the divergence field that will be formed, especially for idealized isolated convection (Figure 9).

Even though some of our simulations ignore conservation laws and are clearly unphysical, the resolution and latent heat and (reference/)ensemble experiments should compare well to realistic uncertainties. Some divergence uncertainty can seemingly be attributed to mode of convection, with embedded processes as underlying causes for differences between these modes of convection. Two mechanisms are thought to potentially govern discrepancy in divergence fields between the squall line simulations and isolated convection cases:

- Recycling of water vapor after condensation (higher fraction in squall line)

- Redistribution of moist static energy by the convective system itself, although it was suggested that this mechanism is statistically indistinguishable or absent

- Yet untracked interactions which take place due to the discrepancy between idealized warm bubble and coldpool forcing

Several other conclusions can be drawn regarding processes affecting the upper air divergence field induced by convective systems:

- Convective momentum mixing is shown to feedback on the convective system via circulation sizes of individual cells and organisation and affects the divergence field.

- The latent heat experiments show that given a certain latent instability, the measure of non-linear response of the divergence profile is depending on factors such as mode of convection.

- Another process that has an important non-linear role is the detrainment of stratospheric air into the troposphere, affecting $\Theta$ and $q_{v}$ fields. This non-linearity is revealed most clearly by MSE redistribution in the experiment where water vapor advection is adjusted. 
Our experiments demonstrate that flow errors can propagate fast from the boundary layer via convective systems and their initialization to near the tropopause, where substantial flow uncertainty may accumulate due to variability and/or misrepresentations of convection. The latter is consistent with Baumgart et al. (2019), where it is shown that the very short term errors are induced ( $<12$ hours) by the convection schemes in ICON and that these subsequently rapidly magnify in the near-tropopause region. Consistently with their results we find that errors caused by uncertainty in the behavior of convective systems can accumulate as divergent flow near the tropopause, which supports that near-tropopause errors (Baumgart et al., 2019) can at least partly relate to earlier convective errors. In addition stratospheric detrainment by mixing of air around convective systems is shown to be able to significantly affect the tropopause region (MSE/energy distribution).

The methods applied here may serve as additional strategies to explain a forecast bust, such as the one documented by Rodwell et al. (2013). In such cases applying a simulation strategy similar to ours to specific convective systems can help to find out whether it is possible to attribute and potentially map the contamination of specific convective systems over the USA to flow uncertainties propagating downstream. Hence, our simulation approach may help to test whether it could generate the correct variability in flow disturbances caused by a (set of) convective cloud(s), so that it could propagate this uncertainty downstream. However, this study may not suggest correct distinctions as the relations in Figure 9 may not be fully reliable.

Code availability. The code used for the experiments and their analysis can be retrieved from:

Author contributions. The basic idea of this study has been developed by HT in collaboration with other co-workers from the TRR 165 . Subsequently this idea was developed further by EG and HT. The simulations have been performed and analysed by EG with contributions from HT. EG also wrote most parts of the manuscript with contributions from HT.

Competing interests. The authors declare that they do not have competing interests.

Acknowledgements. The research leading to these results has been done within the subproject 'A1 - Multiscale analysis of the evolution of forecast uncertainty' of the Transregional Collaborative Research Center SFB / TRR 165 'Waves to Weather' funded by the German Research Foundation (DFG). The initial idea for the performed experiments has emerged during the annual Wavestoweather Meeting 2019 of the Transregional Collaborative Research Center SFB / TRR 165 and was discussed by HT, Ron McTaggart-Cowan, George Craig and Michael Riemer.

The authors want to thank Christopher Polster and Dr. Manuel Baumgartner for their help in setting up CM1 on Mogon2. Parts of this research were conducted using the supercomputer Mogon2 and/or advisory services offered by Johannes Gutenberg University Mainz (hpc.unimainz.de), which is a member of the AHRP (Alliance for High Performance Computing in Rhineland Palatinate, www.ahrp.info) and the 
https://doi.org/10.5194/acp-2020-1142

Preprint. Discussion started: 23 November 2020

(C) Author(s) 2020. CC BY 4.0 License.

(c) (i)

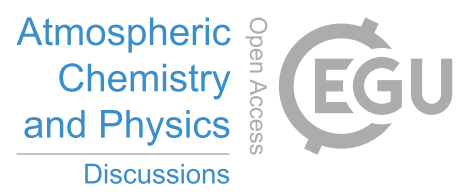

Gauss Alliance e.V. the authors and gratefully acknowledge the computing time granted on the supercomputer Mogon at the JGU Mainz (hpc.uni-mainz.de). 


\section{References}

Arakawa, A.: The Cumulus Parameterization Problem: Past, Present, and Future, JOURNAL OF CLIMATE, 17, $2493-2525,2004$.

Baumgart, M., Ghinassi, P., Wirth, V., Selz, T., Craig, G. C., and Riemer, M.: Quantitative View on the Processes Governing the Upscale Error Growth up to the Planetary Scale Using a Stochastic Convection Scheme, Monthly Weather Review, 147, 1713-1731, https://doi.org/10.1175/mwr-d-18-0292.1, https://doi.org/10.1175/mwr-d-18-0292.1, 2019.

Bierdel, L., Selz, T., and Craig, G.: Theoretical aspects of upscale error growth through the mesoscales: an analytical model, Quarterly Journal of the Royal Meteorological Society, 143, 3048-3059, https://doi.org/10.1002/qj.3160, https://doi.org/10.1002/qj.3160, 2017.

Bierdel, L., Selz, T., and Craig, G. C.: Theoretical aspects of upscale error growth on the mesoscales: Idealized numerical simulations, Quarterly Journal of the Royal Meteorological Society, 144, 682-694, https://doi.org/10.1002/qj.3236, https://doi.org/10.1002/qj.3236, 2018.

Bryan, G.: Cloud Model 1. Version 19.8/cm1r19.8, retrieved/downloaded from: https://www2.mmm.ucar.edu/people/bryan/cml/, 2019.

Bryan, G. H., Wyngaard, J. C., and Fritsch, M. J.: Resolution Requirements for the Simulation of Deep Moist Convection, Monthly Weather Review, 131, 2394-2416, 2003.

Hanley, K. E., Plant, R. S., Stein, T. H. M., Hogan, R. J., Nicol, J. C., Lean, H. W., Halliwell, C., and Clark, P. A.: Mixing-length controls on high-resolution simulations of convective storms, Quarterly Journal of the Royal Meteorological Society, 141, 272-284, https://doi.org/10.1002/qj.2356, 2014.

Hohenegger, C. and Schär, C.: Predictability and Error Growth Dynamics in Cloud-Resolving Models, Journal of the atmospheric sciences, 64, 4467-4478, 2007.

Homeyer, C. R.: Numerical simulations of extratropical tropopause-penetrating convection: Sensitivities to grid resolution, Journal of Geophysical Research: Atmospheres, 120, 7174-7188, https://doi.org/10.1002/2015jd023356, 2015.

Lawson, J. R.: Predictability of Idealized Thunderstorms in Buoyancy-Shear Space, Journal of the Atmospheric Sciences, 76, 2653-2672, https://doi.org/10.1175/jas-d-18-0218.1, 2019.

Markowski, P. and Richardson, Y.: Mesoscale Meteorology in Midlatitudes, Wiley, 2010.

Nuijens, L. and Emanuel, K.: Congestus modes in circulating equilibria of the tropical atmosphere in a two-column model, Quarterly Journal of the Royal Meteorological Society, 144, 2676-2692, https://doi.org/10.1002/qj.3385, https://doi.org/10.1002/qj.3385, 2018.

Petch, J. C.: Sensitivity studies of developing convection in a cloud-resolving model, Quarterly Journal of the Royal Meteorological Society, 132, 345-358, https://doi.org/10.1256/qj.05.71, 2006.

Peters, Karsten, Hohenegger, Cathy, Klocke, and Daniel: Different representation of mesoscale convective systems in convection-permitting and convection-parameterizing NWP models and its implications for large-scale forecast evolution, Atmosphere, https://pure.mpg.de/ pubman/faces/ViewItemOverviewPage.jsp?itemId=item_2570369, 2019.

Rodwell, M. J., Magnusson, L., Bauer, P., Bechtold, P., Bonavita, M., Cardinali, C., Diamantakis, M., Earnshaw, P., Garcia-Mendez, A., Isaksen, L., Källén, E., Klocke, D., Lopez, P., McNally, T., Persson, A., Prates, F., and Wedi, N.: Characteristics of Occasional Poor MediumRange Weather Forecasts for Europe, Bulletin of the American Meteorological Society, 94, 1393-1405, https://doi.org/10.1175/bams-d12-00099.1, https://doi.org/10.1175/bams-d-12-00099.1, 2013.

Selz, T. and Craig, G. C.: Upscale Error Growth in a High-Resolution Simulation of a Summertime Weather Event over Europe, Monthly Weather Review, 143, 813-827, https://doi.org/10.1175/mwr-d-14-00140.1, https://doi.org/10.1175/mwr-d-14-00140.1, 2015a. 
https://doi.org/10.5194/acp-2020-1142

Preprint. Discussion started: 23 November 2020

(C) Author(s) 2020. CC BY 4.0 License.

(c) (i)
Atmospheric

Chemistry

and Physics

Discussions

Selz, T. and Craig, G. C.: Simulation of upscale error growth with a stochastic convection scheme, Geophysical Research Letters, 42, 30563062, https://doi.org/10.1002/2015gl063525, https://doi.org/10.1002/2015g1063525, 2015b.

Stein, T. H. M., Hogan, R. J., Hanley, K. E., Nicol, J. C., Lean, H. W., Plant, R. S., Clark, P. A., and Halliwell, C. E.: The ThreeDimensional Morphology of Simulated and Observed Convective Storms over Southern England, Monthly Weather Review, 142, 3264-3283, https://doi.org/10.1175/mwr-d-13-00372.1, 2014.

Tan, Z.-M., Zhang, F., Rotunno, R., and Snyder, C.: Mesoscale Predictability of Moist Baroclinic Waves: Experiments with Parameterized Convection, Journal of the Atmospheric Sciences, 61, 1794-1804, https://doi.org/10.1175/2007jas2715.1, https://doi.org/10.1175/ 2007 jas2715.1, 2004.

475 Weisman, M. L. and Klemp, J. B.: The Dependence of Numerically Simulated Convective Storms on Vertical Wind Shear and Buoyancy, Monthly Weather Review, 110, 504-520, https://doi.org/10.1175/1520-0493(1982)110<0504:tdonsc>2.0.co;2, https://doi.org/10. 1175/1520-0493(1982)110<0504:tdonsc >2.0.co;2, 1982.

Zhang, F., Bei, N., Rotunno, R., Snyder, C., and Epifanio, C. C.: Mesoscale Predictability of Moist Baroclinic Waves: Convection-Permitting Experiments and Multistage Error Growth Dynamics, Journal of the Atmospheric Sciences, 64, 3579-3594, https://doi.org/10.1175/jas4028.1, https://doi.org/10.1175/jas4028.1, 2007. 